\title{
ASYMPTOTIC BEHAVIOR FOR QUADRATIC VARIATIONS OF NON-GAUSSIAN MULTIPARAMETER HERMITE RANDOM FIELDS
}

BY

\author{
T. T. DIU TRA N* (LUXEMbOURG)
}

\begin{abstract}
Let $\left(Z_{\mathbf{t}}^{q, \mathbf{H}}\right)_{\mathbf{t} \in[0,1]^{d}}$ denote a $d$-parameter Hermite random field of order $q \geqslant 1$ and self-similarity parameter $\mathbf{H}=\left(H_{1}, \ldots, H_{d}\right) \in$ $\left(\frac{1}{2}, 1\right)^{d}$. This process is $\mathbf{H}$-self-similar, has stationary increments and exhibits long-range dependence. Particular examples include fractional Brownian motion $(q=1, d=1)$, fractional Brownian sheet $(q=1, d \geqslant 2)$, the Rosenblatt process $(q=2, d=1)$ as well as the Rosenblatt sheet $(q=2, d \geqslant 2)$. For any $q \geqslant 2, d \geqslant 1$ and $\mathbf{H} \in\left(\frac{1}{2}, 1\right)^{d}$ we show in this paper that a proper renormalization of the quadratic variation of $Z^{q, \mathbf{H}}$ converges in $L^{2}(\Omega)$ to a standard $d$-parameter Rosenblatt random variable with self-similarity index $\mathbf{H}^{\prime \prime}=1+(2 \mathbf{H}-2) / q$.
\end{abstract}

2010 AMS Mathematics Subject Classification: Primary: 60F05, 60H07; Secondary: 60G18, 60H05.

Key words and phrases: Limit theorems, power variations, Hermite random field, Rosenblatt random field, self-similar stochastic processes.

\section{MOTIVATION AND MAIN RESULTS}

In recent years, analyzing the asymptotic behavior of power variations of selfsimilar stochastic processes has attracted a lot of attention. This is because they play an important role in various aspects, both in probability and statistics. As far as quadratic variations are concerned, a classical application is to use them to construct efficient estimators for the self-similarity parameter (see e.g. [2], [15]). For a less conventional application, let us also mention the paper [5], where the authors have used weighted power variations of fractional Brownian motion to compute exact rates of convergence of some approximating schemes associated with one-dimensional fractional stochastic differential equations.

* I thank M. S. Pakkanen and A. Réveillac, the authors of [10], for sharing their paper in progress with me. Another thank goes to my advisor, Professor Ivan Nourdin, for his very careful review of the paper as well as for his comments and corrections. 
In this paper, we deal with the quadratic variation in the context of multiparameter Hermite random fields. To be more specific, let $Z^{q, \mathbf{H}}=\left(Z_{\mathbf{t}}^{q, \mathbf{H}}\right)_{\mathbf{t} \in[0,1]^{d}}$ stand for the $d$-parameter Hermite random field of order $q \geqslant 1$ and self-similarity parameter $\mathbf{H}=\left(H_{1}, \ldots, H_{d}\right) \in\left(\frac{1}{2}, 1\right)^{d}$ (see Definition 2.1 for the precise meaning), and consider a renormalized version of its quadratic variation, namely

$$
V_{\mathbf{N}}:=\frac{1}{\mathbf{N}} \sum_{\mathbf{i}=0}^{\mathbf{N}-1}\left[\mathbf{N}^{2 \mathbf{H}}\left(\Delta Z_{[\mathbf{i} / \mathbf{N},(\mathbf{i}+1) / \mathbf{N}]}^{q, \mathbf{H}}\right)^{2}-1\right],
$$

where $\Delta Z_{[\mathbf{s}, \mathbf{t}]}^{q, \mathbf{H}}$ are the increments of $Z^{q, \mathbf{H}}$ defined as

$$
\Delta Z_{[\mathbf{s}, \mathbf{t}]}^{q, \mathbf{H}}=\sum_{\mathbf{r} \in\{0,1\}^{d}}(-1)^{d-\sum_{i} r_{i}} Z_{\mathbf{s}+\mathbf{r} \cdot(\mathbf{t}-\mathbf{s})}^{q, \mathbf{H}}
$$

and where the bold notation is systematically used in presence of multi-indices (we refer to Section 2 for precise definitions). As illustrating examples, observe that ([L.2) reduces to $\Delta Z_{[s, t]}^{q, H}=Z_{t}^{q, H}-Z_{s}^{q, H}$ when $d=1$, and to $\Delta Z_{[\mathbf{s , t}]}^{q, H_{1}, H_{2}}=$ $Z_{t_{1}, t_{2}}^{q, H_{1}, H_{2}}-Z_{t_{1}, s_{2}}^{q, H_{1}, H_{2}}-Z_{s_{1}, t_{2}}^{q, H_{1}, H_{2}}+Z_{s_{1}, s_{2}}^{q, H_{1}, H_{2}}$ when $d=2$.

It is well known that each Hermite random field $Z^{q, \mathbf{H}}$ is $\mathbf{H}$-self-similar (that is, $\left(Z_{\mathbf{a t}}^{q, \mathbf{H}}\right)_{\mathbf{t} \in \mathbb{R}^{d}} \stackrel{(d)}{=}\left(\mathbf{a}^{\mathbf{H}} Z_{\mathbf{t}}^{q, \mathbf{H}}\right)_{\mathbf{t} \in \mathbb{R}^{d}}$ for any $\mathbf{a}>0$ ), has stationary increments (that is, $\left(\Delta Z_{[0, \mathbf{t}]}^{q, \mathbf{H}}\right)_{\mathbf{t} \in \mathbb{R}^{d}} \stackrel{(d)}{=}\left(\Delta Z_{[\mathbf{h}, \mathbf{h}+\mathbf{t}]}^{q, \mathbf{H}}\right)_{\mathbf{t} \in \mathbb{R}^{d}}$ for all $\left.\mathbf{h} \in \mathbb{R}^{d}\right)$ and exhibits long-range dependence. Also, when $q=1$, observe that $Z^{1, \mathbf{H}}$ is either the fractional Brownian motion (if $d=1$ ) or the fractional Brownian sheet (if $d \geqslant 2$ ); in particular, among all the Hermite random fields $Z^{q, \mathbf{H}}$, it is the only one to be Gaussian. When $q=2$, we use the usual terminology: the Rosenblatt process (if $d=1$ ) or the Rosenblatt sheet (if $d \geqslant 2$ ).

Before describing our results, let us give a brief overview of the current state of the art. Firstly, let us consider the case $q=d=1$, that is, the case where $Z^{1, H}=$ $B^{H}$ is a fractional Brownian motion with Hurst parameter $H$. The behavior of the quadratic variation of $B^{H}$ is well known since the 1980s, and dates back to the seminal works of Breuer and Major [四], Dobrushin and Major [3] , Giraitis and Surgailis [4] or Taqqu [13]. We have, as $N \rightarrow \infty$ :

- If $H<3 / 4$, then

$$
N^{-1 / 2} \sum_{j=1}^{N}\left(N^{2 H}\left(B_{j / N}^{H}-B_{(j-1) / N}^{H}\right)^{2}-1\right) \stackrel{(d)}{\longrightarrow} \mathcal{N}\left(0, \sigma_{H}^{2}\right) .
$$

- If $H=3 / 4$, then

$$
(N \log N)^{-1 / 2} \sum_{j=1}^{N}\left(N^{3 / 2}\left(B_{j / N}^{H}-B_{(j-1) / N}^{H}\right)^{2}-1\right) \stackrel{(d)}{\longrightarrow} \mathcal{N}\left(0, \sigma_{3 / 4}^{2}\right) .
$$


- If $H>3 / 4$, then

$$
N^{1-2 H} \sum_{j=1}^{N}\left(N^{2 H}\left(B_{j / N}^{H}-B_{(j-1) / N}^{H}\right)^{2}-1\right) \stackrel{L^{2}(\Omega)}{\longrightarrow} \text { "Rosenblatt r.v.", }
$$

where "Rosenblatt r.v." denotes the random variable which is the value at time one of the Rosenblatt process.

Secondly, assume now that $q=1$ and $d=2$, that is, consider the case where $Z^{1, \mathbf{H}}$ is a two-parameter fractional Brownian sheet with Hurst parameter $\mathbf{H}=$ $\left(H_{1}, H_{2}\right)$. According to Réveillac et al. [12] and with $\varphi(N, \mathbf{H})$ a suitable scaling factor, the quadratic variation of $Z^{1, \mathbf{H}}$ behaves as follows, as $N \rightarrow \infty$ :

- If $\mathbf{H} \notin(3 / 4,1)^{2}$, then

$$
\varphi(N, \mathbf{H}) \sum_{i=1}^{N} \sum_{j=1}^{N}\left(N^{2 H_{1}+2 H_{2}}\left(\Delta Z_{[(\mathbf{i}-1) / \mathbf{N}, \mathbf{i} / \mathbf{N}]}^{1, \mathbf{H}}\right)^{2}-1\right) \stackrel{(d)}{\longrightarrow} \mathcal{N}\left(0, \sigma_{\mathbf{H}}^{2}\right) .
$$

- If $\mathbf{H} \in(3 / 4,1)^{2}$, then

$$
\begin{array}{r}
\varphi(N, \mathbf{H}) \sum_{i=1}^{N} \sum_{j=1}^{N}\left(N^{2 H_{1}+2 H_{2}}\left(\Delta Z_{[(\mathbf{i}-1) / \mathbf{N}, \mathbf{i} / \mathbf{N}]}^{1, \mathbf{H}}\right)^{2}-1\right) \\
\stackrel{L^{2}(\Omega)}{\longrightarrow} \text { "two-parameter Rosenblatt r.v.", }
\end{array}
$$

where "two-parameter Rosenblatt r.v." means the value at point $\mathbf{1}=(1,1)$ of the two-parameter Rosenblatt sheet.

Here, we observe the following interesting phenomenon: the limit law in the mixture case (that is, when $H_{1} \leqslant 3 / 4$ and $H_{2}>3 / 4$ ) is Gaussian. For simplicity of the exposition, we have only described above what happens when $d=2$. But the asymptotic behavior for the quadratic variation of $Z^{1, \mathbf{H}}$ is actually known for any value of the dimension $d \geqslant 2$, and we refer to Pakkanen and Réveillac [9]-[1]] for precise statements.

Let us finally review the existing literature on the quadratic variation of $Z^{q, \mathbf{H}}$ in the non-Gaussian case, that is, when $q \geqslant 2$. It is certainly because it is a more difficult case to deal with that only the case where $d=1$ has been studied so far. Chronopoulou et al. have shown in [2] (see also [15], [14]) that, properly renormalized, the quadratic variation of $Z^{q, H}$ converges in $L^{2}(\Omega)$, for any $q \geqslant 2$ and any value of $H \in(1 / 2,1)$, to the Rosenblatt random variable. A consequence of this finding is that fractional Brownian motion is the only Hermite process $(d=1)$ for which there exists a range of parameter such that its quadratic variation exhibits normal convergence; indeed, for all the other Hermite processes, it is shown in [2] that we have the convergence toward a non-Gaussian random variable belonging to the second Wiener chaos. 
In the present paper, we study what happens in the remaining cases, that is, when $q$ and $d$ are both greater than or equal to 2. Thanks to our main result, Theorem I.], we have now a complete picture of the asymptotic behavior of the quadratic variation of any Hermite random field.

THEOREM 1.1. Fix $q \geqslant 2, d \geqslant 1$ and $\mathbf{H} \in\left(\frac{1}{2}, 1\right)^{d}$. Let $Z^{q, \mathbf{H}}$ be a d-parameter Hermite random field of order $q$ with self-similarity parameter $\mathbf{H}$ (see Definition (2.]). Then $c_{1, \mathbf{H}}^{-1 / 2} \mathbf{N}^{(2-2 \mathbf{H}) / q}(q ! q)^{-1} V_{\mathbf{N}}$ converges, in $L^{2}(\Omega)$, to the standard $d$-parameter Rosenblatt sheet with self-similarity parameter $1+(2 \mathbf{H}-2) / q$ evaluated at time $\mathbf{1}$, where $c_{1, \mathrm{H}}$ is given by (B.9).

Our proof of Theorem $\mathbb{\|}$ follows a strategy introduced by Tudor and Viens in [15], based on the use of chaotic expansion into multiple Wiener-Itô integrals. Let us sketch it. Since the Hermite random field $Z^{q, \mathbf{H}}$ is an element of the $q$-th Wiener chaos, we can firstly rely on the product formula for multiple integrals to see that the quadratic variation $V_{\mathbf{N}}$ can be decomposed into a sum of multiple integrals of even orders from 2 to $2 q$. Secondly, by using the isometric property of multiple Wiener-Itô integrals and after checking the $L^{2}\left(\left([0,1]^{d}\right)^{2}\right)$ convergence of its kernel, we will prove that the projection onto the second Wiener chaos converges in $L^{2}(\Omega)$ to the $d$-parameter Rosenblatt random variable. Finally, we will check that all the remaining terms in the chaotic expansion are asymptotically negligible.

In conclusion, it is worth pointing out that, irrespective of the self-similarity parameter, the (properly normalized) quadratic variation of any non-Gaussian multiparameter Hermite random fields exhibits a convergence to a random variable belonging to the second Wiener chaos. It is in strong contrast with what happens in the Gaussian case $(q=1)$, where either central or non-central limit theorems may arise, depending on the value of the self-similarity parameter.

The remainder of the paper is structured as follows. Section 2 contains some preliminaries and useful notation. The proof of our main result, Theorem II], is then provided in Section 3.

\section{PRELIMINARIES}

This section describes the notation and the mathematical objects (together with their main properties) that are used throughout this paper.

2.1. Notation. Fix an integer $d \geqslant 1$. In what follows, we shall systematically use bold notation when dealing with multi-indexed quantities. We thus write:

- $\mathbf{a}=\left(a_{1}, a_{2}, \ldots, a_{d}\right)$.

- $\mathbf{a b}=\left(a_{1} b_{1}, a_{2} b_{2}, \ldots, a_{d} b_{d}\right)$ and $\mathbf{a} / \mathbf{b}=\left(a_{1} / b_{1}, a_{2} / b_{2}, \ldots, a_{d} / b_{d}\right)$.

- $[\mathbf{a}, \mathbf{b}]=\prod_{i=1}^{d}\left[a_{i}, b_{i}\right],(\mathbf{a}, \mathbf{b})=\prod_{i=1}^{d}\left(a_{i}, b_{i}\right)$.

- Summation is suppressed as follows: $\sum_{\mathbf{i}=1}^{\mathbf{N}} a_{\mathbf{i}}=\sum_{i_{1}=1}^{N_{1}} \ldots \sum_{i_{d}=1}^{N_{d}} a_{i_{1}, \ldots, i_{d}}$, whereas for products we shall write $\mathbf{a}^{\mathbf{b}}=\prod_{i=1}^{d} a_{i}^{b_{i}}$. Finally, we shall write $\mathbf{a}<\mathbf{b}$ 
(resp. $\mathbf{a} \leqslant \mathbf{b})$ whenever $a_{1}<b_{1}, a_{2}<b_{2}, \ldots, a_{d}<b_{d}$ (resp. $a_{1} \leqslant b_{1}, a_{2} \leqslant b_{2}$, $\left.\ldots, a_{d} \leqslant b_{d}\right)$.

2.2. Multiple Wiener-Itô integrals. We will now briefly review the theory of multiple Wiener-Itô integrals with respect to Brownian sheet, as described e.g. in Nualart's book [8], Chapter 1, or in [9], Section 3. Let $f \in L^{2}\left(\left(\mathbb{R}^{d}\right)^{q}\right)$ and let us denote by $I_{q}^{W}(f)$ the $q$-fold multiple Wiener-Itô integral of $f$ with respect to the standard two-sided Brownian sheet $\left(W_{\mathbf{t}}\right)_{\mathbf{t} \in \mathbb{R}^{d}}$. In symbols, such an integral is written

$$
I_{q}^{W}(f)=\int_{\left(\mathbb{R}^{d}\right)^{q}} f\left(\mathbf{u}_{1}, \ldots, \mathbf{u}_{q}\right) d W_{\mathbf{u}_{1}} \ldots d W_{\mathbf{u}_{q}}
$$

Moreover, one has $I_{q}^{W}(f)=I_{q}^{W}(\widetilde{f})$, where $\widetilde{f}$ is the symmetrization of $f$ defined by the formula

$$
\widetilde{f}\left(\mathbf{u}_{1}, \ldots, \mathbf{u}_{q}\right)=\frac{1}{q !} \sum_{\sigma \in \mathfrak{S}_{q}} f\left(\mathbf{u}_{\sigma(1)}, \ldots, \mathbf{u}_{\sigma(q)}\right)
$$

The set of random variables of the form $I_{q}^{W}(f)$, when $f$ runs over $L^{2}\left(\left(\mathbb{R}^{d}\right)^{q}\right)$, is called the $q$-th Wiener chaos of $W$. Furthermore, if $f \in L^{2}\left(\left(\mathbb{R}^{d}\right)^{p}\right)$ and $g \in$ $L^{2}\left(\left(\mathbb{R}^{d}\right)^{q}\right)$ are two symmetric functions, then

$$
I_{p}^{W}(f) I_{q}^{W}(g)=\sum_{r=0}^{p \wedge q} r !\left(\begin{array}{l}
p \\
r
\end{array}\right)\left(\begin{array}{l}
q \\
r
\end{array}\right) I_{p+q-2 r}^{W}\left(f \widetilde{\otimes}_{r} g\right),
$$

where the contraction $f \otimes_{r} g$, which belongs to $L^{2}\left(\left(\mathbb{R}^{d}\right)^{p+q-2 r}\right)$ for every $r=$ $0,1, \ldots, p \wedge q$, is given by

$$
=\int_{\left(\mathbb{R}^{d}\right)^{r}} f\left(\mathbf{u}_{1}, \ldots, \mathbf{u}_{p-r}, \mathbf{a}_{1}, \ldots, \mathbf{a}_{r}\right) g\left(\mathbf{v}_{1}, \ldots, \mathbf{v}_{q-r}, \mathbf{a}_{1}, \ldots, \mathbf{a}_{r}\right) d \mathbf{a}_{1} \ldots d \mathbf{a}_{r} .
$$

For any $r=0, \ldots, p \wedge q$, the Cauchy-Schwarz inequality yields

$$
\begin{aligned}
\left\|f \widetilde{\otimes}_{r} g\right\|_{L^{2}\left(\left(\mathbb{R}^{d}\right)^{p+q-2 r}\right)} & \leqslant\left\|f \otimes_{r} g\right\|_{L^{2}\left(\left(\mathbb{R}^{d}\right)^{p+q-2 r}\right)} \\
& \leqslant\|f\|_{L^{2}\left(\left(\mathbb{R}^{d}\right)^{p}\right)}\|g\|_{L^{2}\left(\left(\mathbb{R}^{d}\right)^{q}\right)}
\end{aligned}
$$

Also, $f \otimes_{p} g=\langle f, g\rangle_{L^{2}\left(\left(\mathbb{R}^{d}\right)^{p}\right)}$ when $q=p$. Furthermore, the multiple Wiener integrals satisfy the following isometry and orthogonality properties:

$$
E\left[I_{p}^{W}(f) I_{q}^{W}(g)\right]= \begin{cases}p !\langle\widetilde{f}, \widetilde{g}\rangle_{L^{2}\left(\left(\mathbb{R}^{d}\right)^{p}\right)} & \text { if } p=q \\ 0 & \text { if } p \neq q .\end{cases}
$$


2.3. Multiparameter Hermite random fields. Let us now introduce our main object of interest in this paper, the so-called multiparameter Hermite random field. We follow the definition given by Tudor in [14], Chapter 4 .

Definition 2.1. Let $q, d \geqslant 1$ be two integers and let $\mathbf{H}=\left(H_{1}, \ldots, H_{d}\right)$ be a vector belonging to $\left(\frac{1}{2}, 1\right)^{d}$. The $d$-parameter Hermite random field of order $q$ and self-similarity parameter $\mathbf{H}$ is any random field of the form

$$
Z^{q, \mathbf{H}}(\mathbf{t})=c_{q, \mathbf{H}} \int_{\left(\mathbb{R}^{d}\right)^{q}} d W_{u_{1,1}, \ldots, u_{1, d}} \ldots d W_{u_{q, 1}, \ldots, u_{q, d}}
$$

$$
\times\left(\int_{0}^{t_{1}} d a_{1} \ldots \int_{0}^{t_{d}} d a_{d} \prod_{j=1}^{q}\left(a_{1}-u_{j, 1}\right)_{+}^{-\left(1 / 2+\left(1-H_{1}\right) / q\right)} \ldots\left(a_{d}-u_{j, d}\right)_{+}^{-\left(1 / 2+\left(1-H_{d}\right) / q\right)}\right)
$$

$$
=c_{q, \mathbf{H}} \int_{\left(\mathbb{R}^{d}\right)^{q}} d W_{\mathbf{u}_{1}} \ldots d W_{\mathbf{u}_{q}} \int_{0}^{\mathbf{t}} d \mathbf{a} \prod_{j=1}^{q}\left(\mathbf{a}-\mathbf{u}_{j}\right)_{+}^{-(1 / 2+(1-\mathbf{H}) / q)},
$$

where $x_{+}=\max (x, 0), W$ is a standard two-sided Brownian sheet, and $c(q, \mathbf{H})$ is a positive constant depending only on $q$ and $\mathbf{H}$ chosen so that $E\left[Z^{q, \mathbf{H}}(\mathbf{1})^{2}\right]=1$.

The above integral (2.5) represents a multiple Wiener-Itô integral of order $q$ with respect to the standard two-sided Brownian sheet $W$.

In many cases (e.g. when one wants to simulate $Z^{q, \mathbf{H}}$ or when one looks for constructing a stochastic calculus with respect to it), the following finite-time interval representation for $Z^{q, \mathbf{H}}$ may be interested as well:

$$
\begin{aligned}
Z^{q, \mathbf{H}}(\mathbf{t}) \stackrel{(d)}{=} & b_{q, \mathbf{H}} \int_{0}^{t_{1}} \ldots \int_{0}^{t_{d}} d W_{u_{1,1}, \ldots, u_{1, d}} \ldots \int_{0}^{t_{1}} \ldots \int_{0}^{t_{d}} d W_{u_{q, 1}, \ldots, u_{q, d}} \\
& \times\left(\int_{u_{1,1} \vee \ldots \vee u_{q, 1}}^{t_{1}} d a_{1} \partial_{1} K^{H_{1}^{\prime}}\left(a_{1}, u_{1,1}\right) \ldots \partial_{1} K^{H_{1}^{\prime}}\left(a_{1}, u_{q, 1}\right)\right) \ldots \\
& \times\left(\int_{u_{1, d} \vee \ldots \vee u_{q, d}}^{t_{d}} d a_{d} \partial_{1} K^{H_{d}^{\prime}}\left(a_{d}, u_{1, d}\right) \ldots \partial_{1} K^{H_{d}^{\prime}}\left(a_{d}, u_{q, d}\right)\right)
\end{aligned}
$$

$$
=b_{q, \mathbf{H}} \int_{[0, \mathbf{t}]^{q}} d W_{\mathbf{u}_{1}} \ldots d W_{\mathbf{u}_{q}} \prod_{j=1}^{d} \int_{u_{1, j} \vee \ldots \vee u_{q, j}}^{t_{j}} d a \partial_{1} K^{H_{j}^{\prime}}\left(a, u_{1, j}\right) \ldots \partial_{1} K^{H_{j}^{\prime}}\left(a, u_{q, j}\right) .
$$

In (2.6), $K^{H}$ stands for the usual kernel appearing in the classical expression of the fractional Brownian motion $B^{H}$ as a Volterra integral with respect to Brownian motion (see e.g. [6], [四), that is, $B_{t}^{H}=\int_{0}^{t} K^{H}(t, s) d B_{s}$, whereas

$$
b_{q, \mathbf{H}}:=\frac{(\mathbf{H}(2 \mathbf{H}-1))^{1 / 2}}{\left(q !\left(\mathbf{H}^{\prime}\left(2 \mathbf{H}^{\prime}-1\right)\right)^{q}\right)^{1 / 2}}=(\sqrt{q !})^{d-1} \prod_{j=1}^{d} \frac{\left(H_{j}\left(2 H_{j}-1\right)\right)^{1 / 2}}{\left(q !\left(H_{j}^{\prime}\left(2 H_{j}^{\prime}-1\right)\right)^{q}\right)^{1 / 2}}
$$


is the unique positive constant ensuring that $E\left[Z^{q, \mathbf{H}}(\mathbf{1})^{2}\right]=1$, where

$$
\mathbf{H}^{\prime}:=1+\frac{\mathbf{H}-1}{q} \quad\left(\text { iff }\left(2 \mathbf{H}^{\prime}-2\right) q=2 \mathbf{H}-2\right) .
$$

For a proof of (2.6) when $d=2$, we refer to [14], Chapter 4. The extension to any value of $d$ as presented here is straightforward.

\section{PROOF OF THEOREM 1.1}

We are now in a position to give the proof of our Theorem 1.1 . It is divided into three steps.

3.1. Expanding into Wiener chaos. In preparation for analyzing the quadratic

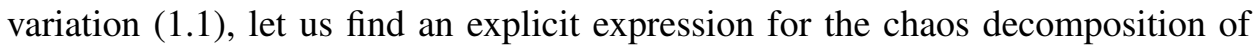
$V_{\mathbf{N}}$. Using (2.6) and proceeding by induction on the dimension $d$, we can write $\Delta Z_{[\mathbf{i} / \mathbf{N},(\mathbf{i}+1) / \mathbf{N}]}^{q, \mathbf{H}}$ as a $q$-th Wiener-Itô integral with respect to the standard twosided Brownian sheet $\left(W_{\mathbf{t}}\right)_{\mathbf{t} \in \mathbb{R}^{d}}$ as follows: for every $0 \leqslant \mathbf{i} \leqslant \mathbf{N}-1$, one has

$$
\Delta Z_{[\mathbf{i} / \mathbf{N},(\mathbf{i}+1) / \mathbf{N}]}^{q, \mathbf{H}}=I_{q}\left(f_{\mathbf{i}, \mathbf{N}}\right),
$$

where

$$
f_{\mathbf{i}, \mathbf{N}}\left(\mathbf{x}_{1}, \ldots, \mathbf{x}_{q}\right)=b_{q, \mathbf{H}} \prod_{j=1}^{d} f_{i_{j}, N_{j}}\left(x_{1, j}, \ldots, x_{q, j}\right),
$$

with $f_{i, N}\left(x_{1}, \ldots, x_{q}\right)$ denoting the expression

$$
\begin{gathered}
\mathbf{1}_{[0,(i+1) / N]}\left(x_{1} \vee \ldots \vee x_{q}\right) \int_{x_{1} \vee \ldots \vee x_{q}}^{(i+1) / N} d u \partial_{1} K^{H^{\prime}}\left(u, x_{1}\right) \ldots \partial_{1} K^{H^{\prime}}\left(u, x_{q}\right) \\
-\mathbf{1}_{[0, i / N]}\left(x_{1} \vee \ldots \vee x_{q}\right) \int_{x_{1} \vee \ldots \vee x_{q}}^{i / N} d u \partial_{1} K^{H^{\prime}}\left(u, x_{1}\right) \ldots \partial_{1} K^{H^{\prime}}\left(u, x_{q}\right)
\end{gathered}
$$

and with $b_{q, \mathbf{H}}$ and $\mathbf{H}^{\prime}$ given by (2.7) and (‥8), respectively. Indeed, for $d=1$, see [2], Section 3, p. 8, it reduces to

$$
\Delta Z_{[i / N,(i+1) / N]}^{q, H}=Z_{(i+1) / N}^{q, H}-Z_{i / N}^{q, H}=b_{q, H} I_{q}\left(f_{i, N}\right),
$$

while for $d=2$ it is easy to verify that

$$
\begin{aligned}
\Delta Z_{[\mathbf{i} / \mathbf{N},(\mathbf{i}+1) / \mathbf{N}]}^{q, \mathbf{H}} & =Z_{(i+1) / N,(j+1) / M}^{q, H_{1}, H_{2}}-Z_{i / N,(j+1) / M}^{q, H_{1}, H_{2}}-Z_{(i+1) / N, j / M}^{q, H_{1}, H_{2}}+Z_{i / N, j / M}^{q, H_{1}, H_{2}} \\
& =I_{q}\left(f_{i, j, N, M}\right),
\end{aligned}
$$


where

$$
\begin{aligned}
& f_{i, j, N, M}\left(x_{1}, y_{1}, \ldots, x_{q}, y_{q}\right) \\
& =b_{q, H_{1}, H_{2}} \mathbf{1}_{[0,(i+1) / N]}\left(x_{1} \vee \ldots \vee x_{q}\right) \int_{x_{1} \vee \ldots \vee x_{q}}^{(i+1) / N} d u \partial_{1} K^{H_{1}^{\prime}}\left(u, x_{1}\right) \ldots \partial_{1} K^{H_{1}^{\prime}}\left(u, x_{q}\right) \\
& \times \mathbf{1}_{[0,(j+1) / M]}\left(y_{1} \vee \ldots \vee y_{q}\right) \int_{y_{1} \vee \ldots \vee y_{q}}^{(j+1) / M} d v \partial_{1} K^{H_{2}^{\prime}}\left(v, y_{1}\right) \ldots \partial_{1} K^{H_{2}^{\prime}}\left(v, y_{q}\right) \\
& -b_{q, H_{1}, H_{2}} \mathbf{1}_{[0,(i+1) / N]}\left(x_{1} \vee \ldots \vee x_{q}\right) \int_{x_{1} \vee \ldots \vee x_{q}}^{(i+1) / N} d u \partial_{1} K^{H_{1}^{\prime}}\left(u, x_{1}\right) \ldots \partial_{1} K^{H_{1}^{\prime}}\left(u, x_{q}\right) \\
& \times \mathbf{1}_{[0, j / M]}\left(y_{1} \vee \ldots \vee y_{q}\right) \int_{y_{1} \vee \ldots \vee y_{q}}^{j / M} d v \partial_{1} K^{H_{2}^{\prime}}\left(v, y_{1}\right) \ldots \partial_{1} K^{H_{2}^{\prime}}\left(v, y_{q}\right) \\
& -b_{q, H_{1}, H_{2}} \mathbf{1}_{[0, i / N]}\left(x_{1} \vee \ldots \vee x_{q}\right) \int_{x_{1} \vee \ldots \vee x_{q}}^{i / N} d u \partial_{1} K^{H_{1}^{\prime}}\left(u, x_{1}\right) \ldots \partial_{1} K^{H_{1}^{\prime}}\left(u, x_{q}\right) \\
& \times \mathbf{1}_{[0,(j+1) / M]}\left(y_{1} \vee \ldots \vee y_{q}\right) \int_{y_{1} \vee \ldots \vee y_{q}}^{(j+1) / M} d v \partial_{1} K^{H_{2}^{\prime}}\left(v, y_{1}\right) \ldots \partial_{1} K^{H_{2}^{\prime}}\left(v, y_{q}\right) \\
& +b_{q, H_{1}, H_{2}} \mathbf{1}_{[0, i / N]}\left(x_{1} \vee \ldots \vee x_{q}\right) \int_{x_{1} \vee \ldots \vee x_{q}}^{i / N} d u \partial_{1} K^{H_{1}^{\prime}}\left(u, x_{1}\right) \ldots \partial_{1} K^{H_{1}^{\prime}}\left(u, x_{q}\right) \\
& \times \mathbf{1}_{[0, j / M]}\left(y_{1} \vee \ldots \vee y_{q}\right) \int_{y_{1} \vee \ldots \vee y_{q}}^{j / M} d v \partial_{1} K^{H_{2}^{\prime}}\left(v, y_{1}\right) \ldots \partial_{1} K^{H_{2}^{\prime}}\left(v, y_{q}\right) \\
& =b_{q, H_{1}, H_{2}} f_{i, N}\left(x_{1}, \ldots, x_{q}\right) f_{j, M}\left(y_{1}, \ldots, y_{q}\right) \text {. }
\end{aligned}
$$

The last equality above is obtained by grouping each term of $f_{i, j, N, M}$ together. Suppose that the expressions (B.1) and (B.2) are true for $d$, that is, the kernel of $\Delta Z_{[\mathbf{i} / \mathbf{N},(\mathbf{i}+1) / \mathbf{N}]}^{q, \mathbf{H}}$ is equal to

$$
\begin{aligned}
b_{q, \mathbf{H}} \sum_{\left(r_{1}, \ldots, r_{d}\right) \in\{0,1\}^{d}}(-1)^{d-\sum_{i=1}^{d} r_{i}} \prod_{j=1}^{d} \mathbf{1}_{\left[0,\left(i_{j}+r_{j}\right) / N_{j}\right]}\left(x_{1, j} \vee \ldots \vee x_{q, j}\right) \\
\quad \times \int_{x_{1, j} \vee \ldots \vee x_{q, j}}^{\left(i_{j}+r_{j}\right) / N_{j}} d u \partial_{1} K^{H_{j}^{\prime}}\left(u, x_{1, j}\right) \ldots \partial_{1} K^{H_{j}^{\prime}}\left(u, x_{q, j}\right) \\
=b_{q, \mathbf{H}} \prod_{j=1}^{d} f_{i_{j}, N_{j}}\left(x_{1, j}, \ldots, x_{q, j}\right) .
\end{aligned}
$$


Then, for the case $d+1$ we have

$$
\begin{aligned}
\Delta Z_{[\mathbf{i} / \mathbf{N},(\mathbf{i}+1) / \mathbf{N}]}^{q, \mathbf{H}}=\sum_{\mathbf{r} \in\{0,1\}^{d+1}}(-1)^{d+1-\sum_{i=1}^{d+1} r_{i}} Z_{(\mathbf{i}+\mathbf{r}) / \mathbf{N}}^{q, \mathbf{H}} & \\
= & \sum_{\left(r_{1}, \ldots, r_{d}\right) \in\{0,1\}^{d}}(-1)^{d-\sum_{i=1}^{d} r_{i}} Z_{\left(\left(i_{1}+r_{1}\right) / N_{1}, \ldots,\left(i_{d}+r_{d}\right) / N_{d},\left(i_{d+1}+1\right) / N_{d+1}\right)}^{q, \mathbf{H}} \\
& +\sum_{\left(r_{1}, \ldots, r_{d}\right) \in\{0,1\}^{d}}(-1)^{d+1-\sum_{i=1}^{d} r_{i}} Z_{\left(\left(i_{1}+r_{1}\right) / N_{1}, \ldots,\left(i_{d}+r_{d}\right) / N_{d}, i_{d+1} / N_{d+1}\right)}^{q, \mathbf{H}} \\
= & \sum_{\left(r_{1}, \ldots, r_{d}\right) \in\{0,1\}^{d}}(-1)^{d-\sum_{i=1}^{d} r_{i}} Z_{\left(\left(i_{1}+r_{1}\right) / N_{1}, \ldots,\left(i_{d}+r_{d}\right) / N_{d},\left(i_{d+1}+1\right) / N_{d+1}\right)}^{q, \mathbf{H}} \\
& -\sum_{\left(r_{1}, \ldots, r_{d}\right) \in\{0,1\}^{d}}(-1)^{d-\sum_{i=1}^{d} r_{i}} Z_{\left(\left(i_{1}+r_{1}\right) / N_{1}, \ldots,\left(i_{d}+r_{d}\right) / N_{d}, i_{d+1} / N_{d+1}\right)}^{q, \mathbf{H}} .
\end{aligned}
$$

It belongs to the $q$-th Wiener chaos with the kernel $f_{\mathbf{i}, \mathbf{N}}$ given by

$$
\begin{aligned}
f_{\mathbf{i}, \mathbf{N}}= & b_{q, \mathbf{H}} \sum_{\left(r_{1}, \ldots, r_{d}\right) \in\{0,1\}^{d}}(-1)^{d-\sum_{i=1}^{d} r_{i}} \prod_{j=1}^{d} \mathbf{1}_{\left.\left[0, i_{j}+r_{j}\right) / N_{j}\right]}\left(x_{1, j} \vee \ldots \vee x_{q, j}\right) \\
& \times \int_{x_{1, j} \vee \ldots \vee x_{q, j}}^{\left(i_{j}+r_{j}\right) / N_{j}} d u \partial_{1} K^{H_{j}^{\prime}}\left(u, x_{1, j}\right) \ldots \partial_{1} K^{H_{j}^{\prime}}\left(u, x_{q, j}\right) \\
& \times \int_{x_{1, d+1} \vee \ldots \vee x_{q, d+1}}^{\left(i_{d+1}+1\right) / N_{d+1}} d u^{\prime} \partial_{1} K^{H_{d+1}^{\prime}}\left(u^{\prime}, x_{1, d+1}\right) \ldots \partial_{1} K^{H_{d+1}^{\prime}}\left(u^{\prime}, x_{q, d+1}\right) \\
& \left.-\int_{x_{1, d+1} \vee \ldots \vee x_{q, d+1}}^{i_{d+1} / N_{d+1}} d u^{\prime} \partial_{1} K^{H_{d+1}^{\prime}}\left(u^{\prime}, x_{1, d+1}\right) \ldots \partial_{1} K^{H_{d+1}^{\prime}}\left(u^{\prime}, x_{q, d+1}\right)\right) .
\end{aligned}
$$

By induction, one gets $f_{\mathbf{i}, \mathbf{N}}=b_{q, \mathbf{H}} \prod_{j=1}^{d+1} f_{i_{j}, N_{j}}\left(x_{1, j}, \ldots, x_{q, j}\right)$, which is our desired expression.

$$
\left(\Delta Z_{[\mathbf{i} / \mathbf{N},(\mathbf{i}+1) / \mathbf{N}]}^{q, \mathbf{H}}\right)^{2}-E\left[\left(\Delta Z_{[\mathbf{i} / \mathbf{N},(\mathbf{i}+1) / \mathbf{N}]}^{q, \mathbf{H}}\right)^{2}\right]=\sum_{r=0}^{q-1} r !\left(\begin{array}{c}
q \\
r
\end{array}\right)^{2} I_{2 q-2 r}\left(f_{\mathbf{i}, \mathbf{N}} \widetilde{\otimes}_{r} f_{\mathbf{i}, \mathbf{N}}\right) .
$$

Let us compute the contractions appearing on the right-hand side of (3.4). For every $0 \leqslant r \leqslant q-1$, we have

$$
\begin{aligned}
\left(f_{\mathbf{i}, \mathbf{N}} \otimes_{r}\right. & \left.f_{\mathbf{i}, \mathbf{N}}\right)\left(\mathbf{x}_{1}, \ldots, \mathbf{x}_{2 q-2 r}\right) \\
= & \int_{\left([0,1]^{d}\right)^{r}} d \mathbf{a}_{1} \ldots d \mathbf{a}_{r} f_{\mathbf{i}, \mathbf{N}}\left(\mathbf{x}_{1}, \ldots, \mathbf{x}_{q-r}, \mathbf{a}_{1}, \ldots, \mathbf{a}_{r}\right) \\
& \times f_{\mathbf{i}, \mathbf{N}}\left(\mathbf{x}_{q-r+1}, \ldots, \mathbf{x}_{2 q-2 r}, \mathbf{a}_{1}, \ldots, \mathbf{a}_{r}\right)
\end{aligned}
$$




$$
\begin{aligned}
= & b_{q, \mathbf{H}}^{2} \int_{\left([0,1]^{d}\right)^{r}} d \mathbf{a}_{1} \ldots d \mathbf{a}_{r} \prod_{j=1}^{d} f_{i_{j}, N_{j}}\left(x_{1, j}, \ldots, x_{q-r, j}, a_{1, j}, \ldots, a_{r, j}\right) \\
& \times \prod_{j=1}^{d} f_{i_{j}, N_{j}}\left(x_{q-r+1, j}, \ldots, x_{2 q-2 r, j}, a_{1, j}, \ldots, a_{r, j}\right) \\
= & b_{q, \mathbf{H}}^{2} \prod_{j=1}^{d}\left(f_{i_{j}, N_{j}} \otimes_{r} f_{i_{j}, N_{j}}\right)\left(x_{1, j}, \ldots, x_{2 q-2 r, j}\right),
\end{aligned}
$$

where

$$
\begin{aligned}
& \text { (3.6) }\left(f_{i, N} \otimes_{r} f_{i, N}\right)\left(x_{1}, \ldots, x_{2 q-2 r}\right)=\left(H^{\prime}\left(2 H^{\prime}-1\right)\right)^{r} \\
& \times\left\{\mathbf{1}_{[0,(i+1) / N]}\left(x_{1} \vee \ldots \vee x_{q-r}\right) \int_{x_{1} \vee \ldots \vee x_{q-r}}^{(i+1) / N} d u \partial_{1} K^{H^{\prime}}\left(u, x_{1}\right) \ldots \partial_{1} K^{H^{\prime}}\left(u, x_{q-r}\right)\right. \\
& \times \mathbf{1}_{[0,(i+1) / N]}\left(x_{q-r+1} \vee \ldots \vee x_{2 q-2 r}\right) \int_{x_{q-r+1} \vee \ldots \vee x_{2 q-2 r}}^{(i+1) / N} d u^{\prime} \partial_{1} K^{H^{\prime}}\left(u^{\prime}, x_{q-r+1}\right) \ldots \\
& \ldots \partial_{1} K^{H^{\prime}}\left(u^{\prime}, x_{2 q-2 r}\right)\left|u-u^{\prime}\right|^{\left(2 H^{\prime}-2\right) r} \\
& -\mathbf{1}_{[0,(i+1) / N]}\left(x_{1} \vee \ldots \vee x_{q-r}\right) \int_{x_{1} \vee \ldots \vee x_{q-r}}^{(i+1) / N} d u \partial_{1} K^{H^{\prime}}\left(u, x_{1}\right) \ldots \partial_{1} K^{H^{\prime}}\left(u, x_{q-r}\right) \\
& \times \mathbf{1}_{[0, i / N]}\left(x_{q-r+1} \vee \ldots \vee x_{2 q-2 r}\right) \int_{x_{q-r+1} \vee \ldots \vee x_{2 q-2 r}}^{i / N} d u^{\prime} \partial_{1} K^{H^{\prime}}\left(u^{\prime}, x_{q-r+1}\right) \ldots \\
& \ldots \partial_{1} K^{H^{\prime}}\left(u^{\prime}, x_{2 q-2 r}\right)\left|u-u^{\prime}\right|^{\left(2 H^{\prime}-2\right) r} \\
& -\mathbf{1}_{[0, i / N]}\left(x_{1} \vee \ldots \vee x_{q-r}\right) \int_{x_{1} \vee \ldots \vee x_{q-r}}^{i / N} d u \partial_{1} K^{H^{\prime}}\left(u, x_{1}\right) \ldots \partial_{1} K^{H^{\prime}}\left(u, x_{q-r}\right) \\
& \times \mathbf{1}_{[0,(i+1) / N]}\left(x_{q-r+1} \vee \ldots \vee x_{2 q-2 r}\right) \int_{x_{q-r+1} \vee \ldots \vee x_{2 q-2 r}}^{(i+1) / N} d u^{\prime} \partial_{1} K^{H^{\prime}}\left(u^{\prime}, x_{q-r+1}\right) \ldots \\
& \ldots \partial_{1} K^{H^{\prime}}\left(u^{\prime}, x_{2 q-2 r}\right)\left|u-u^{\prime}\right|^{\left(2 H^{\prime}-2\right) r} \\
& +\mathbf{1}_{[0, i / N]}\left(x_{1} \vee \ldots \vee x_{q-r}\right) \int_{x_{1} \vee \ldots \vee x_{q-r}}^{i / N} d u \partial_{1} K^{H^{\prime}}\left(u, x_{1}\right) \ldots \partial_{1} K^{H^{\prime}}\left(u, x_{q-r}\right) \\
& \times \mathbf{1}_{[0, i / N]}\left(x_{q-r+1} \vee \ldots \vee x_{2 q-2 r}\right) \int_{x_{q-r+1} \vee \ldots \vee x_{2 q-2 r}}^{i / N} d u^{\prime} \partial_{1} K^{H^{\prime}}\left(u^{\prime}, x_{q-r+1}\right) \ldots \\
& \left.\ldots \partial_{1} K^{H^{\prime}}\left(u^{\prime}, x_{2 q-2 r}\right)\left|u-u^{\prime}\right|^{\left(2 H^{\prime}-2\right) r}\right\} .
\end{aligned}
$$

(See [2], p. 10, for a detailed computation of the expression ([3.6).) Moreover, since 
$Z^{q, \mathbf{H}}$ is $\mathbf{H}$-self-similar and has stationary increments, we obtain

$$
\Delta Z_{[\mathbf{i} / \mathbf{N},(\mathbf{i}+1) / \mathbf{N}]}^{q, \mathbf{H}} \stackrel{(d)}{=} \mathbf{N}^{-\mathbf{H}} \Delta Z_{[\mathbf{i}, \mathbf{i}+1]}^{q, \mathbf{H}} \stackrel{(d)}{=} \mathbf{N}^{-\mathbf{H}} Z_{[0, \mathbf{1}]}^{q, \mathbf{H}} .
$$

It follows that

$$
E\left[\mathbf{N}^{2 \mathbf{H}}\left(\Delta Z_{[\mathbf{i} / \mathbf{N},(\mathbf{i}+1) / \mathbf{N}]}^{q, \mathbf{H}}\right)^{2}\right]=E\left[Z^{q, \mathbf{H}}(\mathbf{1})^{2}\right]=1
$$

As a consequence, we have

$$
V_{\mathbf{N}}=F_{2 q, \mathbf{N}}+c_{2 q-2} F_{2 q-2, \mathbf{N}}+\ldots+c_{4} F_{4, \mathbf{N}}+c_{2} F_{2, \mathbf{N}},
$$

where $c_{2 q-2 r}=r !\left(\begin{array}{l}q \\ r\end{array}\right)^{2}, r=0, \ldots, q-1$, are the combinational constants coming from the product formula, and

$$
F_{2 q-2 r, \mathbf{N}}:=\mathbf{N}^{2 \mathbf{H}-1} I_{2 q-2 r}\left(\sum_{\mathbf{i}=0}^{\mathbf{N}-1} f_{\mathbf{i}, \mathbf{N}} \widetilde{\otimes}_{r} f_{\mathbf{i}, \mathbf{N}}\right)
$$

for the kernels $f_{\mathbf{i}, \mathbf{N}} \widetilde{\otimes}_{r} f_{\mathbf{i}, \mathbf{N}}$ computed in ([3.5)).

3.2. Evaluating the $L^{2}(\Omega)$-norm. Set

$$
\begin{aligned}
c_{1, \mathbf{H}}= & \frac{2 ! 2^{d} b_{q, \mathbf{H}}^{4}\left(\mathbf{H}^{\prime}\left(2 \mathbf{H}^{\prime}-1\right)\right)^{2 q}}{\left(4 \mathbf{H}^{\prime}-3\right)\left(4 \mathbf{H}^{\prime}-2\right)\left[\left(2 \mathbf{H}^{\prime}-2\right)(q-1)+1\right]^{2}\left[\left(\mathbf{H}^{\prime}-1\right)(q-1)+1\right]^{2}} \\
= & \frac{(q !)^{2(d-1)}}{2^{d-1}} \prod_{j=1}^{d} \frac{4\left(H_{j}\left(2 H_{j}-1\right)\right)^{2}}{(q !)^{2}\left(4 H_{j}^{\prime}-3\right)\left(4 H_{j}^{\prime}-2\right)} \\
& \times \prod_{j=1}^{d} \frac{1}{\left[\left(2 H_{j}^{\prime}-2\right)(q-1)+1\right]^{2}\left[\left(H_{j}^{\prime}-1\right)(q-1)+1\right]^{2}} .
\end{aligned}
$$

We claim that

$$
\lim _{\mathbf{N} \rightarrow \infty} E\left[c_{1, \mathbf{H}^{-1}}^{-1} \mathbf{N}^{2\left(2-2 \mathbf{H}^{\prime}\right)} c_{2}^{-2} V_{\mathbf{N}}^{2}\right]=1
$$

Let us prove (B.10). Due to the orthogonality property for Wiener chaos of different orders, it is sufficient to evaluate the $L^{2}(\Omega)$-norm of each multiple WienerItô integral appearing in the chaotic decomposition (B.7) of $V_{\mathrm{N}}$. Let us start with the double integral:

$$
F_{2, \mathbf{N}}=\mathbf{N}^{2 \mathbf{H}-1} I_{2}\left(\sum_{\mathbf{i}=0}^{\mathbf{N}-1} f_{\mathbf{i}, \mathbf{N}} \otimes_{q-1} f_{\mathbf{i}, \mathbf{N}}\right) .
$$


Since the kernel $\sum_{\mathbf{i}=0}^{\mathbf{N}-1} f_{\mathbf{i}, \mathbf{N}} \otimes_{q-1} f_{\mathbf{i}, \mathbf{N}}$ is symmetric, we have

$$
\begin{aligned}
E\left[F_{2, \mathbf{N}}^{2}\right] & =2 N^{4 \mathbf{H}-2}\left\|\sum_{\mathbf{i}=0}^{\mathbf{N}-1} f_{\mathbf{i}, \mathbf{N}} \otimes_{q-1} f_{\mathbf{i}, \mathbf{N}}\right\|_{L^{2}\left(\left([0,1]^{d}\right)^{2}\right)}^{2} \\
& =2 \mathbf{N}^{4 \mathbf{H}-2} \sum_{\mathbf{i}, \mathbf{k}=0}^{\mathbf{N}-1}\left\langle f_{\mathbf{i}, \mathbf{N}} \otimes_{q-1} f_{\mathbf{i}, \mathbf{N}}, f_{\mathbf{k}, \mathbf{N}} \otimes_{q-1} f_{\mathbf{k}, \mathbf{N}}\right\rangle_{L^{2}\left(\left([0,1]^{d}\right)^{2}\right)}
\end{aligned}
$$

Let us now compute the scalar products in the above expression. By using (B.5), we get

$$
\begin{aligned}
& \left\langle f_{\mathbf{i}, \mathbf{N}} \otimes_{q-1} f_{\mathbf{i}, \mathbf{N}}, f_{\mathbf{k}, \mathbf{N}} \otimes_{q-1} f_{\mathbf{k}, \mathbf{N}}\right\rangle_{L^{2}\left(\left([0,1]^{d}\right)^{2}\right)} \\
= & b_{q, \mathbf{H}}^{4} \prod_{j=1}^{d}\left\langle f_{i_{j}, N_{j}} \otimes_{q-1} f_{i_{j}, N_{j}}, f_{k_{j}, N_{j}} \otimes_{q-1} f_{k_{j}, N_{j}}\right\rangle_{L^{2}\left([0,1]^{d}\right)} \\
= & b_{q, \mathbf{H}}^{4}\left(\mathbf{H}^{\prime}\left(2 \mathbf{H}^{\prime}-1\right)\right)^{2 q} \prod_{j=1}^{d} \int_{i_{j} / N_{j}}^{\left(i_{j}+1\right) / N_{j}} d u_{j} \int_{i_{j} / N_{j}}^{\left(i_{j}+1\right) / N_{j}} d v_{j} \int_{k_{j} / N_{j}}^{\left(k_{j}+1\right) / N_{j}} d u_{j}^{\prime} \int_{k_{j} / N_{j}}^{\left(k_{j}+1\right) / N_{j}} d v_{j}^{\prime} \\
& \times\left|u_{j}-v_{j}\right|^{\left(2 H_{j}^{\prime}-2\right)(q-1)}\left|u_{j}^{\prime}-v_{j}^{\prime}\right|^{\left(2 H_{j}^{\prime}-2\right)(q-1)}\left|u_{j}-u_{j}^{\prime}\right|^{2 H_{j}^{\prime}-2}\left|v_{j}-v_{j}^{\prime}\right|^{2 H_{j}^{\prime}-2} .
\end{aligned}
$$

The change of variables $u^{\prime}=(u-i / N) N$ for each $u_{j}, u_{j}^{\prime}, v_{j}, v_{j}^{\prime}$ with $j$ from 1 to $d$ yields

$$
\begin{aligned}
& E\left[F_{2, \mathbf{N}}^{2}\right]=2 b_{q, \mathbf{H}}^{4}\left(\mathbf{H}^{\prime}\left(2 \mathbf{H}^{\prime}-1\right)\right)^{2 q} \mathbf{N}^{4 \mathbf{H}-2} \mathbf{N}^{-4} \mathbf{N}^{-\left(2 \mathbf{H}^{\prime}-2\right) 2 q} \\
& \times \sum_{\mathbf{i}, \mathbf{k}=0}^{\mathbf{N}-1} \prod_{j=1}^{d} \int_{0}^{1} d u_{j} \int_{0}^{1} d v_{j} \int_{0}^{1} d u_{j}^{\prime} \int_{0}^{1} d v_{j}^{\prime}\left|u_{j}-v_{j}\right|^{\left(2 H_{j}^{\prime}-2\right)(q-1)}\left|u_{j}^{\prime}-v_{j}^{\prime}\right|^{\left(2 H_{j}^{\prime}-2\right)(q-1)} \\
& \times\left|u_{j}-u_{j}^{\prime}+i_{j}-k_{j}\right|^{2 H_{j}^{\prime}-2}\left|v_{j}-v_{j}^{\prime}+i_{j}-k_{j}\right|^{2 H_{j}^{\prime}-2} .
\end{aligned}
$$

We split the sum $\sum_{\mathbf{i}, \mathbf{k}=0}^{\mathbf{N}-1}$ appearing in $E\left[F_{2, \mathbf{N}}^{2}\right]$ just above into

$$
\sum_{\mathbf{i}, \mathbf{k}=0}^{\mathbf{N}-1}=\sum_{\substack{\mathbf{i}, \mathbf{k}=0 \\ \exists 1 \leqslant j \leqslant d: i_{j}=k_{j}}}^{\mathbf{N}-1}+\sum_{\substack{\mathbf{i}, \mathbf{k}=0 \\ \forall j: i_{j} \neq k_{j}}}^{\mathbf{N}-1} .
$$

For the first term, without loss of generality, we can assume that $i_{1}=k_{1}$ and $i_{j} \neq$ $k_{j}$ for all $j \neq 1$. Then, 


$$
\begin{aligned}
\mathbf{N}^{-2} & \sum_{\substack{\mathbf{i}, \mathbf{k}=0 \\
i_{1}=k_{1}}}^{\mathbf{N}-1} \prod_{j=1}^{d} \int_{[0,1]^{4}} d u_{j} d v_{j} d u_{j}^{\prime} d v_{j}^{\prime}\left|u_{j}-v_{j}\right|^{\left(2 H_{j}^{\prime}-2\right)(q-1)}\left|u_{j}^{\prime}-v_{j}^{\prime}\right|^{\left(2 H_{j}^{\prime}-2\right)(q-1)} \\
& \times\left|u_{j}-u_{j}^{\prime}+i_{j}-k_{j}\right|^{2 H_{j}^{\prime}-2}\left|v_{j}-v_{j}^{\prime}+i_{j}-k_{j}\right|^{2 H_{j}^{\prime}-2} \\
= & N_{1}^{-1} \int_{[0,1]^{4}} d u_{1} d v_{1} d u_{1}^{\prime} d v_{1}^{\prime}\left|u_{1}-v_{1}\right|^{\left(2 H_{1}^{\prime}-2\right)(q-1)}\left|u_{1}^{\prime}-v_{1}^{\prime}\right|^{\left(2 H_{1}^{\prime}-2\right)(q-1)} \\
& \times\left|u_{1}-u_{1}^{\prime}\right|^{2 H_{1}^{\prime}-2}\left|v_{1}-v_{1}^{\prime}\right|^{2 H_{1}^{\prime}-2} \\
& \times \prod_{j=2}^{d} \int_{[0,1]^{4}} d u_{j} d v_{j} d u_{j}^{\prime} d v_{j}^{\prime}\left|u_{j}-v_{j}\right|^{\left(2 H_{j}^{\prime}-2\right)(q-1)}\left|u_{j}^{\prime}-v_{j}^{\prime}\right|^{\left(2 H_{j}^{\prime}-2\right)(q-1)} \\
& \times 2 N_{j}^{-2} \sum_{i_{j}, k_{j}=0}^{N_{j}-1}\left|u_{j}-u_{j}^{\prime}+i_{j}-k_{j}\right|^{2 H_{j}^{\prime}-2}\left|v_{j}-v_{j}^{\prime}+i_{j}-k_{j}\right|^{2 H_{j}^{\prime}-2} .
\end{aligned}
$$

Following [2] or [15], we see that

$$
\begin{aligned}
& N^{-2} \sum_{\substack{i, k=0 \\
i>k}}^{N-1}\left|u-u^{\prime}+i-k\right|^{2 H^{\prime}-2}\left|v-v^{\prime}+i-k\right|^{2 H^{\prime}-2} \\
& =N^{2\left(2 H^{\prime}-2\right)} \frac{1}{N} \sum_{n=1}^{N}\left(1-\frac{n}{N}\right)\left|\frac{u-u^{\prime}}{N}+\frac{n}{N}\right|^{2 H^{\prime}-2}\left|\frac{v-v^{\prime}}{N}+\frac{n}{N}\right|^{2 H^{\prime}-2}
\end{aligned}
$$

is asymptotically equivalent to

$$
N^{2\left(2 H^{\prime}-2\right)} \int_{0}^{1}(1-x) x^{4 H^{\prime}-4} d x=N^{2\left(2 H^{\prime}-2\right)} \frac{1}{\left(4 H^{\prime}-3\right)\left(4 H^{\prime}-2\right)} .
$$

It follows that

$$
\begin{aligned}
& \mathbf{N}^{-2} \sum_{\substack{\mathbf{i}, \mathbf{k}=0 \\
i_{1}=k_{1}}}^{\mathbf{N}-1} \prod_{j=1}^{d} \int_{[0,1]^{4}} d u_{j} d v_{j} d u_{j}^{\prime} d v_{j}^{\prime}\left|u_{j}-v_{j}\right|^{\left(2 H_{j}^{\prime}-2\right)(q-1)}\left|u_{j}^{\prime}-v_{j}^{\prime}\right|^{\left(2 H_{j}^{\prime}-2\right)(q-1)} \\
& \times\left|u_{j}-u_{j}^{\prime}+i_{j}-k_{j}\right|^{2 H_{j}^{\prime}-2}\left|v_{j}-v_{j}^{\prime}+i_{j}-k_{j}\right|^{2 H_{j}^{\prime}-2} \\
& \approx N_{1}^{-1} \int_{[0,1]^{4}} d u_{1} d v_{1} d u_{1}^{\prime} d v_{1}^{\prime}\left|u_{1}-v_{1}\right|^{\left(2 H_{1}^{\prime}-2\right)(q-1)}\left|u_{1}^{\prime}-v_{1}^{\prime}\right|^{\left(2 H_{1}^{\prime}-2\right)(q-1)} \\
& \times\left|u_{1}-u_{1}^{\prime}\right|^{2 H_{1}^{\prime}-2}\left|v_{1}-v_{1}^{\prime}\right|^{2 H_{1}^{\prime}-2} \prod_{j=2}^{d} 2 N_{j}^{2\left(2 H_{j}^{\prime}-2\right)} \\
& \times \frac{1}{\left(4 H_{j}^{\prime}-3\right)\left(4 H_{j}^{\prime}-2\right)\left[\left(2 H_{j}^{\prime}-2\right)(q-1)+1\right]^{2}\left[\left(H_{j}^{\prime}-1\right)(q-1)+1\right]^{2}}
\end{aligned}
$$


Similarly for the second term, where $i_{j} \neq k_{j}$ for all $1 \leqslant j \leqslant d$, we have

$$
\begin{aligned}
& \mathbf{N}^{-2} \sum_{\substack{\mathbf{i}, \mathbf{k}=0 \\
\forall j: i_{j} \neq k_{j}}}^{\mathbf{N}-1} \prod_{j=1}^{d} \int_{[0,1]^{4}} d u_{j} d v_{j} d u_{j}^{\prime} d v_{j}^{\prime}\left|u_{j}-v_{j}\right|^{\left(2 H_{j}^{\prime}-2\right)(q-1)}\left|u_{j}^{\prime}-v_{j}^{\prime}\right|^{\left(2 H_{j}^{\prime}-2\right)(q-1)} \\
& \times\left|u_{j}-u_{j}^{\prime}+i_{j}-k_{j}\right|^{2 H_{j}^{\prime}-2}\left|v_{j}-v_{j}^{\prime}+i_{j}-k_{j}\right|^{2 H_{j}^{\prime}-2} \\
& \approx \prod_{j=1}^{d} 2 N_{j}^{2\left(2 H_{j}^{\prime}-2\right)} \\
& \times \frac{1}{\left(4 H_{j}^{\prime}-3\right)\left(4 H_{j}^{\prime}-2\right)\left[\left(2 H_{j}^{\prime}-2\right)(q-1)+1\right]^{2}\left[\left(H_{j}^{\prime}-1\right)(q-1)+1\right]^{2}}
\end{aligned}
$$

Putting everything together, we conclude that

$$
\lim _{\mathbf{N} \rightarrow \infty} E\left[c_{1, \mathbf{H}}^{-1} \mathbf{N}^{2\left(2-2 \mathbf{H}^{\prime}\right)} F_{2, \mathbf{N}}^{2}\right]=1
$$

Let us now consider the remaining terms $F_{4, \mathbf{N}}, \ldots, F_{2 q, \mathbf{N}}$ in the chaos decomposition (3.7). Since $\|\widetilde{g}\|_{L^{2}} \leqslant\|g\|_{L^{2}}$ for any square integrable function $g$, one can write, for every $0 \leqslant r \leqslant q-2$,

$$
\begin{aligned}
E\left[F_{2 q-2 r, \mathbf{N}}^{2}\right] & =\mathbf{N}^{4 \mathbf{H}-2}(2 q-2 r) !\left\|\sum_{\mathbf{i}=0}^{\mathbf{N}-1} f_{\mathbf{i}, \mathbf{N}} \widetilde{\otimes}_{r} f_{\mathbf{i}, \mathbf{N}}\right\|_{L^{2}\left(\left([0,1]^{d}\right)^{2 q-2 r}\right)}^{2} \\
& \leqslant \mathbf{N}^{4 \mathbf{H}-2}(2 q-2 r) !\left\|\sum_{\mathbf{i}=0}^{\mathbf{N}-1} f_{\mathbf{i}, \mathbf{N}} \otimes_{r} f_{\mathbf{i}, \mathbf{N}}\right\|_{L^{2}\left(\left([0,1]^{d}\right)^{2 q-2 r}\right)}^{2} \\
& =(2 q-2 r) ! \mathbf{N}^{4 \mathbf{H}-2} \sum_{\mathbf{i}, \mathbf{k}=0}^{\mathbf{N}-1}\left\langle f_{\mathbf{i}, \mathbf{N}} \otimes_{r} f_{\mathbf{i}, \mathbf{N}}, f_{\mathbf{k}, \mathbf{N}} \otimes_{r} f_{\mathbf{k}, \mathbf{N}}\right\rangle_{L^{2}\left(\left([0,1]^{d}\right)^{2 q-2 r}\right)}
\end{aligned}
$$

Proceeding as above, we obtain

$$
\begin{aligned}
& \left.\left.\left\langle f_{\mathbf{i}, \mathbf{N}} \otimes_{r} f_{\mathbf{i}, \mathbf{N}}, f_{\mathbf{k}, \mathbf{N}} \otimes_{r} f_{\mathbf{k}, \mathbf{N}}\right\rangle_{L^{2}([0,1]}\right]^{d \cdot(2 q-2 r)}\right) \\
= & b_{q, \mathbf{H}}^{4}\left(\mathbf{H}^{\prime}\left(2 \mathbf{H}^{\prime}-1\right)\right)^{2 q} \prod_{j=1}^{d} \int_{i_{j} / N_{j}}^{\left(i_{j}+1\right) / N_{j}} d u_{j} \int_{i_{j} / N_{j}}^{\left(i_{j}+1\right) / N_{j}} d v_{j} \int_{k_{j} / N_{j}}^{\left(k_{j}+1\right) / N_{j}} d u_{j}^{\prime} \int_{k_{j} / N_{j}}^{\left(k_{j}+1\right) / N_{j}} d v_{j}^{\prime} \\
\times & \left|u_{j}-v_{j}\right|^{\left(2 H_{j}^{\prime}-2\right) r}\left|u_{j}^{\prime}-v_{j}^{\prime}\right|^{\left(2 H_{j}^{\prime}-2\right) r}\left|u_{j}-u_{j}^{\prime}\right|^{\left.\left(2 H_{j}^{\prime}-2\right)(q-r)\right)}\left|v_{j}-v_{j}^{\prime}\right|^{\left(2 H_{j}^{\prime}-2\right)(q-r)} .
\end{aligned}
$$

Using the change of variables $u^{\prime}=(u-i / N) N$ for each $u_{j}, u_{j}, v_{j}, v_{j}^{\prime}$ with $j=$ $1, \ldots, d$, we get 


$$
\begin{aligned}
& E\left[F_{2 q-2 r, \mathbf{N}}^{2}\right] \\
\leqslant & (2 q-2 r) ! b_{q, \mathbf{H}}^{4}\left(\mathbf{H}^{\prime}\left(2 \mathbf{H}^{\prime}-1\right)\right)^{2 q} \mathbf{N}^{4 \mathbf{H}-2} \mathbf{N}^{-4} \mathbf{N}^{-\left(2 \mathbf{H}^{\prime}-2\right) 2 q} \\
& \times \sum_{\mathbf{i}, \mathbf{k}=0}^{\mathbf{N}-1} \prod_{j=1}^{d} \int_{0}^{1} d u_{j} \int_{0}^{1} d v_{j} \int_{0}^{1} d u_{j}^{\prime} \int_{0}^{1} d v_{j}^{\prime}\left|u_{j}-v_{j}\right|^{\left(2 H_{j}^{\prime}-2\right) r}\left|u_{j}^{\prime}-v_{j}^{\prime}\right|^{\left(2 H_{j}^{\prime}-2\right) r} \\
& \times\left|u_{j}-u_{j}^{\prime}+i_{j}-k_{j}\right|^{\left(2 H_{j}^{\prime}-2\right)(q-r)}\left|v_{j}-v_{j}^{\prime}+i_{j}-k_{j}\right|^{\left(2 H_{j}^{\prime}-2\right)(q-r)} \\
= & (2 q-2 r) ! b_{q, \mathbf{H}}^{4}\left(\mathbf{H}^{\prime}\left(2 \mathbf{H}^{\prime}-1\right)\right)^{2 q} \mathbf{N}^{-2} \\
& \times \prod_{j=1}^{d} \int_{[0,1]} d u_{j} d v_{j} d u_{j}^{\prime} d v_{j}^{\prime}\left|u_{j}-v_{j}\right|^{\left(2 H_{j}^{\prime}-2\right) r}\left|u_{j}^{\prime}-v_{j}^{\prime}\right|^{\left(2 H_{j}^{\prime}-2\right) r} \\
& \times \sum_{i_{j}, k_{j}=0}^{N_{j}-1}\left|u_{j}-u_{j}^{\prime}+i_{j}-k_{j}\right|^{\left(2 H_{j}^{\prime}-2\right)(q-r)}\left|v_{j}-v_{j}^{\prime}+i_{j}-k_{j}\right|^{\left(2 H_{j}^{\prime}-2\right)(q-r)} \\
= & (2 q-2 r) ! b_{q, \mathbf{H}}^{4}\left(\mathbf{H}^{\prime}\left(2 \mathbf{H}^{\prime}-1\right)\right)^{2 q} \mathbf{N}^{2\left(2 \mathbf{H}^{\prime}-2\right)(q-r)} \\
& \times \prod_{j=1}^{d} \int_{[0,1]} d u_{j} d v_{j} d u_{j}^{\prime} d v_{j}^{\prime}\left|u_{j}-v_{j}\right|^{\left(2 H_{j}^{\prime}-2\right) r}\left|u_{j}^{\prime}-v_{j}^{\prime}\right|^{\left(2 H_{j}^{\prime}-2\right) r} \\
& \times \frac{2}{N_{j}} \sum_{n_{j}=1}^{N_{j}}\left(1-\frac{n_{j}}{N_{j}}\right)\left|\frac{u_{j}-u_{j}^{\prime}}{N_{j}}+\frac{n_{j}}{N_{j}}\right|^{\left(2 H_{j}^{\prime}-2\right)(q-r)}\left|\frac{v_{j}-v_{j}^{\prime}}{N_{j}}+\frac{n_{j}}{N_{j}}\right|^{\left(2 H_{j}^{\prime}-2\right)(q-r)} .
\end{aligned}
$$

Apart from the diagonal terms, the term $\left(u-u^{\prime}\right) / N$ is dominated by $n / N$ for $n, N$ large enough. Using a Riemann sum approximation, one has for $0 \leqslant r \leqslant q-2$ and as $\mathbf{N} \rightarrow \infty$,

$$
E\left[\mathbf{N}^{\left(2-2 \mathbf{H}^{\prime}\right)(2 q-2 r)} F_{2 q-2 r, \mathbf{N}}^{2}\right]=O(1) .
$$

We deduce that

$$
\lim _{\mathbf{N} \rightarrow \infty} E\left[\mathbf{N}^{2\left(2-2 \mathbf{H}^{\prime}\right)} F_{2 q-2 r, \mathbf{N}}^{2}\right]=0,
$$

and the proof of (B.10) follows.

3.3. Concluding the proof of Theorem 1.1. Thanks to (3.10), to understand the asymptotic behavior of the renormalized sequence of $V_{\mathrm{N}}$, it is enough to analyze the convergence of the term

$$
I_{2}\left(\mathbf{N}^{2 \mathbf{H}-1} \mathbf{N}^{2-2 \mathbf{H}^{\prime}} \sum_{\mathbf{i}=0}^{\mathbf{N}-1} f_{\mathbf{i}, \mathbf{N}} \otimes_{q-1} f_{\mathbf{i}, \mathbf{N}}\right)
$$

with

$$
f_{\mathbf{i}, \mathbf{N}} \otimes_{q-1} f_{\mathbf{i}, \mathbf{N}}\left(\mathbf{x}_{1}, \mathbf{x}_{2}\right)=b_{q, \mathbf{H}}^{2} \prod_{j=1}^{d}\left(f_{i_{j}, N_{j}} \otimes_{q-1} f_{i_{j}, N_{j}}\right)\left(x_{1, j}, x_{2, j}\right)
$$




$$
\begin{aligned}
& =b_{q, \mathbf{H}}^{2}\left(\mathbf{H}^{\prime}\left(2 \mathbf{H}^{\prime}-1\right)\right)^{q-1} \\
& \times \prod_{j=1}^{d}\left(\mathbf{1}_{\left[0, i_{j} / N_{j}\right]}\left(x_{1, j}\right) \mathbf{1}_{\left[0, i_{j} / N_{j}\right]}\left(x_{2, j}\right) \int_{i_{j} / N_{j}}^{\left(i_{j}+1\right) / N_{j}} d u \int_{i_{j} / N_{j}}^{\left(i_{j}+1\right) / N_{j}} d u^{\prime} \partial_{1} K^{H_{j}^{\prime}}\left(u, x_{1, j}\right)\right. \\
& \times \partial_{1} K^{H_{j}^{\prime}}\left(u^{\prime}, x_{2, j}\right)\left|u-u^{\prime}\right|^{\left(2 H_{j}^{\prime}-2\right)(q-1)} \\
& +\mathbf{1}_{\left[0, i_{j} / N_{j}\right]}\left(x_{1, j}\right) \mathbf{1}_{\left[i_{j} / N_{j},\left(i_{j}+1\right) / N_{j}\right]}\left(x_{2, j}\right) \int_{i_{j} / N_{j}}^{\left(i_{j}+1\right) / N_{j}} d u \int_{x_{2, j}}^{\left(i_{j}+1\right) / N_{j}} d u^{\prime} \partial_{1} K^{H_{j}^{\prime}}\left(u, x_{1, j}\right) \\
& \times \partial_{1} K^{H_{j}^{\prime}}\left(u^{\prime}, x_{2, j}\right)\left|u-u^{\prime}\right|^{\left(2 H_{j}^{\prime}-2\right)(q-1)} \\
& +\mathbf{1}_{\left[i_{j} / N_{j},\left(i_{j}+1\right) / N_{j}\right]}\left(x_{1, j}\right) \mathbf{1}_{\left[0,\left(i_{j}+1\right) / N_{j}\right]}\left(x_{2, j}\right) \int_{x_{1, j}}^{\left(i_{j}+1\right) / N_{j}} d u \\
& \times \int_{i_{j} / N_{j}}^{\left(i_{j}+1\right) / N_{j}} d u^{\prime} \partial_{1} K^{H_{j}^{\prime}}\left(u, x_{1, j}\right) \partial_{1} K^{H_{j}^{\prime}}\left(u^{\prime}, x_{2, j}\right)\left|u-u^{\prime}\right|^{\left(2 H_{j}^{\prime}-2\right)(q-1)} \\
& +\mathbf{1}_{\left[i_{j} / N_{j}, i_{j} / N_{j}\right]}\left(x_{1, j}\right) \mathbf{1}_{\left[i_{j} / N_{j},\left(i_{j}+1\right) / N_{j}\right]}\left(x_{2, j}\right) \int_{x_{1, j}}^{\left(i_{j}+1\right) / N_{j}} d u \\
& \left.\times \int_{x_{2, j}}^{\left(i_{j}+1\right) / N_{j}} d u^{\prime} \partial_{1} K^{H_{j}^{\prime}}\left(u, x_{1, j}\right) \partial_{1} K^{H_{j}^{\prime}}\left(u^{\prime}, x_{2, j}\right)\left|u-u^{\prime}\right|^{\left(2 H_{j}^{\prime}-2\right)(q-1)}\right) .
\end{aligned}
$$

Among the four terms of each factor on the right-hand side of the above expression, only the first one is not asymptotically negligible in $L^{2}(\Omega)$, see [2], pp. 14 and 15, or follow the lines of [15] for details. Furthermore, by the isometry property for multiple Wiener-Itô integrals, evaluating the $L^{2}(\Omega)$-limit of a sequence belonging to the second Wiener chaos is equivalent to evaluating the $L^{2}\left(\left([0,1]^{d}\right)^{2}\right)$-limit of the sequence of their corresponding symmetric kernels. Therefore, we are left to find the limit of $f_{2}^{\mathbf{N}}$ in $L^{2}\left(\left([0,1]^{d}\right)^{2}\right)$, where

$$
\begin{aligned}
& f_{2}^{\mathbf{N}}\left(\mathbf{x}_{1}, \mathbf{x}_{2}\right):=(q !)^{d-1} \prod_{j=1}^{d} \frac{H_{j}\left(2 H_{j}-1\right)}{q !\left(H_{j}^{\prime}\left(2 H_{j}^{\prime}-1\right)\right)^{q}}\left(H_{j}^{\prime}\left(2 H_{j}^{\prime}-1\right)\right)^{q-1} N_{j}^{2 H_{j}-1} N_{j}^{2-2 H_{j}^{\prime}} \\
& \times \sum_{1_{j}=0}^{N_{j}-1} \mathbf{1}_{\left[0, i_{j} / N_{j}\right]}\left(x_{1, j}\right) \mathbf{1}_{\left[0, i_{j} / N_{j}\right]}\left(x_{2, j}\right) \int_{i_{j} / N_{j}}^{\left(i_{j}+1\right) / N_{j}} d u \int_{i_{j} / N_{j}}^{\left(i_{j}+1\right) / N_{j}} d u^{\prime} \partial_{1} K^{H_{j}^{\prime}}\left(u, x_{1, j}\right) \\
& \times \partial_{1} K^{H_{j}^{\prime}}\left(u^{\prime}, x_{2, j}\right)\left|u-u^{\prime}\right|^{\left(2 H_{j}^{\prime}-2\right)(q-1)} .
\end{aligned}
$$

According to [2], Theorem 3.2, by using integral approximation and the Lebesgue dominated convergence theorem, each term under the product for $j$ from 1 to $d$ converges in $L^{2}\left([0,1]^{2}\right)$ to the constant $a_{j}$ times the kernel of a standard Rosenblatt 
random variable $Z^{2,2 H_{j}^{\prime}-1}(1)$ as $N_{j} \rightarrow \infty$ with $a_{j}=\frac{2 H_{j}\left(2 H_{j}-1\right)}{q !\left(4 H_{j}^{\prime}-3\right)^{1 / 2}\left(4 H_{j}^{\prime}-2\right)^{1 / 2}\left[\left(2 H_{j}^{\prime}-2\right)(q-1)+1\right]\left[\left(H_{j}^{\prime}-1\right)(q-1)+1\right]}$.

Therefore, $f_{2}^{\mathbf{N}}$ converges in $L^{2}\left(\left([0,1]^{d}\right)^{2}\right)$ to the constant $2^{(d-1) / 2} c_{1, \mathbf{H}}^{1 / 2}$ times the kernel of a standard Rosenblatt sheet at time $\mathbf{1}$ as $\mathbf{N} \rightarrow \infty$, which leads to our desired conclusion.

\section{REFERENCES}

[1] P. Breuer and P. Major, Central limit theorems for nonlinear functionals of Gaussian fields, J. Multivariate Anal. 13 (3) (1983), pp. 425-441.

[2] A. Chronopoulou, C. A. Tudor, and F. G. Viens, Self-similarity parameter estimation and reproduction property for non-Gaussian Hermite processes, Commun. Stoch. Anal. 5 (1) (2011), Article 10.

[3] R. L. Dobrushin and P. Major, Non-central limit theorems for nonlinear functionals of Gaussian fields, Z. Wahrsch. Verw. Gebiete 50 (1) (1979), pp. 27-52.

[4] L. Giraitis and D. Surgailis, CLT and other limit theorems for functionals of Gaussian processes, Z. Wahrsch. Verw. Gebiete 70 (2) (1985), pp. 191-212.

[5] M. Gradinaru and I. Nourdin, Milstein's type schemes for fractional SDEs, Ann. Inst. Henri Poincaré Probab. Stat. 45 (4) (2009), pp. 1085-1098.

[6] I. Nourdin, Asymptotic behavior of weighted quadratic and cubic variations of fractional Brownian motion, Ann. Probab. 36 (6) (2008), pp. 2159-2175.

[7] I. Nourdin, Selected Aspects of Fractional Brownian Motion, Springer and Bocconi University Press, Milan 2012.

[8] D. Nualart, The Malliavin Calculus and Related Topics, second edition, Springer, Berlin 2006.

[9] M. S. Pakkanen and A. Réveillac, Functional limit theorems for generalized variations of the fractional Brownian sheet, Bernoulli 22 (3) (2016), pp. 1671-1708.

[10] M. S. Pakkanen and A. Réveillac, Functional limit theorems for weighted quadratic variations of fractional Brownian sheets, in preparation.

[11] A. Réveillac, Convergence of finite-dimensional laws of the weighted quadratic variations process for some fractional Brownian sheets, Stoch. Anal. Appl. 27 (1) (2009), pp. 51-73.

[12] A. Réveillac, M. Stauch, and C. A. Tudor, Hermite variations of the fractional Brownian sheet, Stoch. Dyn. 12 (3) (2012), 1150021.

[13] M. S. Taqqu, Convergence of integrated processes of arbitrary Hermite rank, Z. Wahrsch. Verw. Gebiete 50 (1) (1979), pp. 53-83.

[14] C. A. Tudor, Analysis of Variations for Self-similar Processes: A Stochastic Calculus Approach, Springer, Cham 2013.

[15] C. A. Tudor and F. G. Viens, Variations and estimators for self-similarity parameters via Malliavin calculus, Ann. Probab. 37 (6) (2009), pp. 2093-2134.

T. T. Diu Tran

Université du Luxembourg, UR en Mathématiques

6, avenue de la Fonte

L-4364 Esch-sur-Alzette, Luxembourg

E-mail: ttdiu.tran@gmail.com 